\title{
Distributed Hybrid Precoding for Indoor Deployments Using Millimeter Wave Band
}

\author{
Sonia Gimenez, Daniel Calabuig, Sandra Roger, Jose F. Monserrat, and Narcís Cardona \\ iTEAM Research Institute, Universitat Politècnica de València, 46022 Valencia, Spain \\ Correspondence should be addressed to Jose F. Monserrat; jomondel@iteam.upv.es
}

Received 18 April 2017; Accepted 27 August 2017; Published 8 October 2017

Academic Editor: Massimo Condoluci

Copyright (C) 2017 Sonia Gimenez et al. This is an open access article distributed under the Creative Commons Attribution License, which permits unrestricted use, distribution, and reproduction in any medium, provided the original work is properly cited.

Distributed Antenna Systems (DAS) are an alternative of network deployment that allows reducing the distance between transmitter and receiver by distributing the antennas throughout the coverage area. Moreover, the performance of the millimeter wave (mmWave) band can be significantly high within short transmitter-receiver distances. In this paper, the potential benefits of DAS deployments in the mmWave band are studied. To this aim, a distributed hybrid precoding (DHP) solution with remote antenna unit (RAU) selection capabilities is proposed and analyzed in an indoor DAS working in mmWaves and compared to two other indoor deployment strategies: a conventional cellular system with colocated antenna arrays and a small cell deployment. The results show that, using DHP, DAS not only brings huge gains to cell-edge users rate but also increases system capacity, becoming the best overall deployment. Further simulations including practical limitations have revealed that DAS using DHP is quite robust to combiner losses, although its performance is significantly degraded by outdated channel reports.

\section{Introduction}

In order to address the huge data rate demands and high connectivity and mobility required by next-generation mobile networks, a deep change in the cellular architecture design is needed. Especially for indoor users, which represent approximately $80 \%$ of the total traffic conveyed by a network, the definition of new deployment strategies is essential $[1,2]$. Together with the use of small cells, Distributed Antenna Systems (DAS) have been proposed as a promising deployment strategy to improve coverage and capacity in large indoor zones [3].

DAS are network architectures in which the antenna elements of a Base Station (BS) are geographically distributed throughout the coverage area. The main advantage of this approach is that the average distance from any point of the scenario to the nearest antenna is reduced [4]. Due to this fact, the link budget, coverage, and outage probability can be in most cases improved. Although the deployment of small cells can be more flexible and scalable, the use of DAS allows a simpler coordination among the different remote antenna units (RAUs), due to the high-bandwidth and low-latency dedicated connection between antennas and BSs, such as wires, fiber optics, or Radio Frequency (RF) links [5]. In general, these connections can be implemented in either a passive or an active form [6]. On one hand, in active DAS deployments, not only the antennas but also the radio units are distributed over the space. Besides, active components are incorporated into the fronthaul to compensate the losses of the distribution system. On the other hand, in passive DAS deployments, only passive components are used to distribute the signals over the space. Due to this, passive DAS is a costeffective solution for medium-sized distances [7], where the losses of the distribution system are not significant, being the most used approach indoors [6].

DAS transmission schemes that select the antenna with lowest path loss reduce the total network transmission power and increase the battery life. Furthermore, lower transmission power reduces the interference level and, hence, increases the system capacity. However, these schemes cannot benefit from the natural RAUs cooperation ability of DAS. In fact, DAS can be viewed as a multiantenna system, where the antennas are not colocated with the BS [5]. In order to make the most of the Multiple-Input Multiple-Output (MIMO) channel richness, more advanced Single-User (SU) MIMO and Multiuser (MU) MIMO techniques can be applied to DAS. Furthermore, path 
loss differences between the distributed antennas become significant in DAS, so techniques selecting only a subset of the antennas for transmission can bring large benefits to the system [8]. The performance of SU and MU MIMO techniques in DAS with different degrees of cooperation among RAUs is analyzed in $[4,9]$. Both works conclude that large performance gains are achieved when antenna selection schemes are applied.

In parallel to the advances on network architectures, the use of the mmWave band has been also spotted as a promising technology for indoor communications due to several reasons [1]. Besides the advantage of having large bandwidth availability at this frequency band, defined from 30 to $300 \mathrm{GHz}$, the large penetration losses caused by the external surfaces of the buildings make indoor scenarios be well isolated from outdoor interference, while, at the same time, indoor materials are shown to introduce relatively low losses [10]. However, the application of baseband MIMO precoding techniques in mmWave systems with large antennas is limited by the high cost and power consumption introduced by the components of the RF chains performing the upconversion of the baseband signals to these higher frequencies (low noise amplifier, mixer, and analog-to-digital converter) [11], making a solution with as many RF chains as antennas in the BSs a very impractical approach. Motivated by this, hybrid transmission architectures are being investigated by the research community, where the precoding process is divided between the analog (RF) and digital (baseband) domains, alleviating the number of required RF chains of the system [12]. Several works analyze the performance of this hybrid architecture in different scenarios $[13,14]$. However, the topic of DAS hybrid precoding in mmWave has not previously been addressed in the literature, to the best of the authors' knowledge.

It is worth noting that the joint utilization of DAS and mmWaves for indoor scenarios can bring about large benefits of synergy, thanks to the reduction of the distance between transmitter and receiver given by DAS and to the optimum performance of mmWaves within short links. This motivates the work presented in this paper, in which the performance of DAS working in mmWaves has been assessed. To this aim, a distributed hybrid precoding solution with RAU selection capabilities is proposed for indoor DAS deployments, and its performance is analyzed and compared to two other indoor deployment strategies: a conventional cellular system with colocated antenna arrays and a small cell deployment. System level simulations are used to assess the benefits and drawbacks of DAS working in mmWave band, showing that our technique outperforms other state-of-theart solutions. Simulation results are presented considering both ideal and nonideal simulation assumptions in order to better understand the impact of two important practical limitations of the hybrid architectures: the use of outdated Channel State Information (CSI) at the transmitter and the losses introduced by nonideal RF combiners.

The following notations are used throughout this paper. The boldface capital letter $\mathbf{X}$ and the boldface small letter $\mathbf{x}$ denote a matrix and a vector, respectively; a small italic letter $x$ denotes a scalar; $\mathbb{C}$ represents the set of complex numbers;
$\|\mathbf{X}\|_{\mathrm{F}}$ denotes the Frobenius norm of $\mathbf{X} ;|\mathbf{x}|$ represents the modulus of $\mathbf{x}$. The transpose and Hermitian of $\mathbf{X}$ are represented by $\mathbf{X}^{\top}$ and $\mathbf{X}^{\dagger}$, respectively, and $\operatorname{tr}\{\mathbf{X}\}$ is the trace of $\mathrm{X}$.

The rest of the paper is organized as follows. Sections 2 and 3 describe the fundamentals of the hybrid architecture and the precoding algorithms utilized, respectively. In Section 4, the distributed hybrid precoding (DHP) scheme for DAS is presented, and Section 5 details the simulation setup and deployment configuration. Section 6 presents the discussion of results under both ideal and nonideal conditions and, finally, the main findings of the paper are drawn in Section 7.

\section{System Model}

This section details the system model, including the description of the DAS deployment and the hybrid architecture considered in this paper for the application of hybrid precoding in mmWaves.

Let us consider a cellular network with $N_{\text {BS }}$ BSs implementing a hybrid transmission scheme [11] and $N_{\mathrm{UE}}$ User Equipment devices (UEs), where each BS serves $K$ users: $K N_{\mathrm{BS}}=N_{\mathrm{UE}}$. This model is depicted in Figure 1, where each BS is equipped with $N_{t}^{R F}$ RF chains and $M$ antennas, while at the UE, the number of RF chains and antennas is given by $N_{\mathrm{r}}^{\mathrm{RF}}$ and $N$, respectively. This architecture is considered for deployments with distributed antennas as well as for deployments with colocated antennas. In the former case, we assume a passive DAS deployment, in which only the antennas are distributed over the space, whereas all other components of the BS are centralized. We also assume that, in DAS deployment, the $M$ antennas of the BS are distributed in $R$ RAUs consisting of $M_{R}=M / R$ antennas each. Moreover, antennas are connected to the RF unit outputs through ideal coaxial wires. In the case of colocated antennas, the $M$ antennas of the BS are located together with the rest of BS elements.

The first stage of the preprocessing at the BS corresponds to the user selection process, where $N_{s}$ out of the $K$ users are selected to be served: $N_{s} \leq N_{\mathrm{t}}^{\mathrm{RF}}$. Assuming that only a single data stream is transmitted to each user, the vector of data symbols to be transmitted by one BS at each time instant, $\mathbf{s} \in \mathbb{C}^{N_{s} \times 1}$, can be expressed as

$$
\mathbf{s}=\left[s_{1}, \ldots, s_{N_{s}}\right]^{\top}
$$

where $s_{l}, l=1, \ldots, N_{s}$, are the independent symbols with equal power to be transmitted to the selected users. Then, preprocessing at baseband is applied by means of the matrix $\mathbf{B}_{\mathrm{BB}} \in \mathbb{C}^{N_{\mathrm{t}}^{\mathrm{RF}} \times N_{s}}$, using any kind of linear precoding technique satisfying $\operatorname{tr}\left\{\mathbf{B}_{\mathrm{BB}}\left(\mathbf{B}_{\mathrm{BB}}\right)^{\dagger}\right\}=1$. The last stage of the data preprocessing is performed at RF, that is, after the upconversion of the signals, when beamforming is applied by means of phase shifters and combiners. A set of $M$ phase shifters is applied to the output of each RF chain. Afterwards, the outputs of the $i$ th phase shifters of every RF chain are combined to feed the $i$ th antenna element in the array. As a result of this process, $N_{t}^{R F}$ different beams are conformed in 


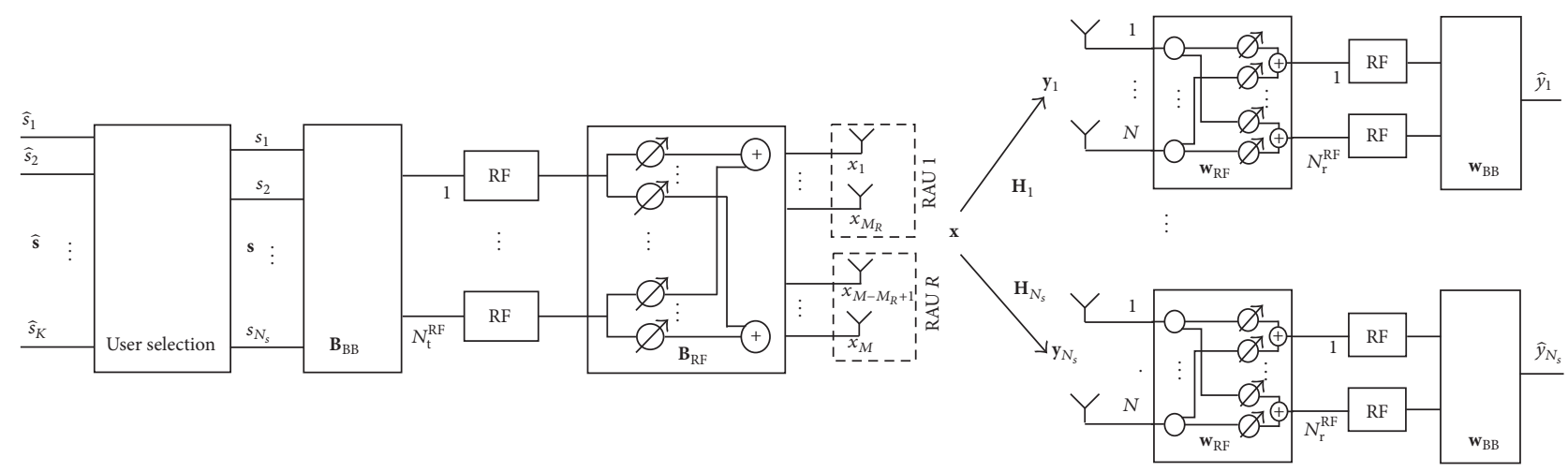

FIGURE 1: Hybrid precoding transmission model for indoor scenarios.

order to transmit the RF signals. This process can be modeled by means of an $M \times N_{\mathrm{t}}^{\mathrm{RF}}$ complex matrix, $\mathbf{B}_{\mathrm{RF}}$. In particular, the data vector $\mathbf{x} \in \mathbb{C}^{M \times 1}$ transmitted by the BS can be expressed as

$$
\mathbf{x}=\mathbf{B}_{\mathrm{RF}} \mathbf{B}_{\mathrm{BB}} \mathbf{s} .
$$

In order to limit the power transmitted by the antennas, power normalization is applied to $\mathbf{x}$ in such a way that $\operatorname{tr}\left\{\mathbb{E}\left[\mathbf{x x}^{\dagger}\right]\right\}=P$, with $P$ being the total available power per BS.

In a multicell scenario and using the superscript $(l)$ to denote that a variable is related to BS $l$, the received signal at the $u$ th user served by BS $i$ can be expressed as

$$
\mathbf{y}_{u, i}=\sum_{l=1}^{N_{\mathrm{BS}}} \mathbf{H}_{u, i}^{(l)} \mathbf{x}^{(l)}+\mathbf{n},
$$

where $\mathbf{H}_{u, i}^{(l)} \in \mathbb{C}^{N \times M}$ is the MIMO channel matrix between BS $l$ and the $u$ th UE served by BS $i, \mathbf{x}^{(l)}$ is the data vector transmitted by BS $l$, and $\mathbf{n} \in \mathbb{C}^{N \times 1}$ is the Additive White Gaussian Noise (AWGN) with zero mean and covariance $\mathbb{E}\left[\mathbf{n n}^{\dagger}\right]=\sigma_{n}^{2} \mathbf{I}_{N}$, where $\mathbf{I}_{N}$ is the $N \times N$ identity matrix.

Using (2) and (3), the Signal to Interference plus Noise Ratio (SINR) for the $u$ th UE served by BS $i$ before the RF and baseband processing at the receiver, $\operatorname{SINR}_{u, i}$, can be expressed as

$$
\begin{aligned}
& \operatorname{SINR}_{u, i} \\
& =\frac{P\left|\mathbf{H}_{u, i}^{(i)} \mathbf{B}_{\mathrm{RF}}^{(i)} \mathbf{B}_{\mathrm{BB}, u}^{(i)}\right|^{2}}{P\left|\mathbf{H}_{u, i}^{(i)} \mathbf{B}_{\mathrm{RF}}^{(i)} \sum_{k \neq u}^{N_{s}} \mathbf{B}_{\mathrm{BB}, k}^{(i)}\right|^{2}+P\left\|\sum_{l \neq i}^{N_{\mathrm{Bs}}} \mathbf{H}_{u, i}^{(l)} \mathbf{B}_{\mathrm{RF}}^{(l)} \mathbf{B}_{\mathrm{BB}}^{(l)}\right\|_{\mathrm{F}}^{2}+\sigma_{n}^{2}},
\end{aligned}
$$

where $\mathbf{B}_{\mathrm{BB}, k}^{(i)}$ denotes the $k$ th column of the matrix $\mathbf{B}_{\mathrm{BB}}^{(i)}$.

\section{MIMO Precoding Techniques}

In this section, the state-of-the-art MIMO precoding techniques used in this paper to evaluate the performance of different indoor deployment strategies are briefly described.

For the sake of simplicity, both the number of antennas, $N$, and the number of RF chains at the receiver, $N_{\mathrm{RF}}$, are assumed to be 1 hereinafter.
3.1. Fully Digital Precoding. Fully digital precoding is the simplest and optimum approach when the number of available $\mathrm{RF}$ chains at the transmitter, $N_{\mathrm{t}}^{\mathrm{RF}}$, is equal to the number of antennas, $M$. In this case, data processing is applied to only the baseband signal, without further modifications after the conversion to RF. Mathematically, this is equivalent to $\mathbf{B}_{\mathrm{RF}}=\mathbf{I}_{M}$, and, thus, (2) becomes

$$
\mathbf{x}=\mathbf{B}_{\mathrm{BB}} \mathbf{s}
$$

In multicell scenarios, Regularized Zero Forcing (RZF) has been proven to provide good results when compared to other digital linear precoding techniques $[15,16]$, since this technique also considers the impact of the background noise and unknown user interference originated by the neighbor cells [17]. RZF precoding matrix computed by BS $i$ is shown in the following equation:

$$
\begin{aligned}
\widetilde{\mathbf{B}}_{\mathrm{BB}}^{(i), \mathrm{RZF}} & =\mathbf{H}^{(i)^{\dagger}}\left(\mathbf{H}^{(i)} \mathbf{H}^{(i)^{\dagger}}+\alpha \mathbf{I}\right)^{-1}, \\
\alpha_{\mathrm{RZF}}^{(i)} & =\frac{1}{\sqrt{\operatorname{tr}\left\{\widetilde{\mathbf{B}}_{\mathrm{BB}}^{(i), \mathrm{RZF}}\left(\widetilde{\mathbf{B}}_{\mathrm{BB}}^{(i), \mathrm{RZF}}\right)^{\dagger}\right\}}}, \\
\mathbf{B}_{\mathrm{BB}}^{(i), \mathrm{RZF}} & =\alpha_{\mathrm{RZF}}^{(i)} \widetilde{\mathbf{B}}_{\mathrm{BB}}^{(i), \mathrm{RZF}},
\end{aligned}
$$

where $\mathbf{H}^{(i)} \in \mathbb{C}^{N_{s} \times M}$ is the channel matrix between the BS $i$ and its $N_{s}$ users being served, given by

$$
\mathbf{H}^{(i)}=\left[\begin{array}{llll}
\mathbf{h}_{1, i}^{(i)} & \mathbf{h}_{2, i}^{(i)} & \cdots & \mathbf{h}_{N_{s}, i}^{(i)}
\end{array}\right]^{\top},
$$

where $\mathbf{h}_{u, i}^{(i)} \in \mathbb{C}^{M \times 1}, u=1, \ldots, N_{s}, \mathbf{I} \in \mathbb{C}^{N_{s} \times N_{s}}$ is the identity matrix, and $\alpha=K \sigma_{n}^{2}$ to maximize SINR [17]. Note that $\mathbf{B}_{\mathrm{BB}}^{\mathrm{RZF}}$ is the baseband linear precoder that will be used along this paper.

3.2. RF Beamforming. RF beamforming reduces the number of required RF chains at the BS and consequently the cost and power consumption of the mixed analog/digital signal components [12] of the RF units, what makes this kind of precoding interesting for mmWaves, where cost and power consumption are design limitations. Since the number of 
applicable precoding vectors in RF beamforming is already limited by hardware to a finite set, the use of predefined codebooks becomes a good strategy to reduce the amount of feedback information required by the system.

Several precoding codebooks are available in the literature [18]. Due to its simplicity and effectiveness for uniform linear antenna arrays as shown in [19], DFT codebook is often preferred to more complex designs. The $c$ th codeword of a DFT-based codebook is

$$
\mathbf{w}_{c}^{\mathrm{DFT}}=\frac{1}{\sqrt{M}}\left[1, e^{j 2 \pi c / C}, \ldots, e^{j 2 \pi c / C(M-1)}\right]^{\top},
$$

where $C$ is the number of codewords in the codebook. If $C=M$, it can be shown that the maximum diversity order is guaranteed. The beamforming vector for the $u$ th user served by $\mathrm{BS} i$, denoted as $\mathbf{b}_{u, i}^{\mathrm{DFT}}$, is chosen as

$$
\begin{aligned}
\mathbf{b}_{u, i}^{\mathrm{DFT}} & =\mathbf{w}_{c^{*}}^{\mathrm{DFT}}, \\
c^{*} & =\arg \max _{c}\left(\left|\mathbf{h}_{u, i}^{(i)^{\top}} \mathbf{w}_{c}^{\mathrm{DFT}}\right|^{2}\right) .
\end{aligned}
$$

Note that (9) maximizes the Signal to Noise Ratio (SNR) at the receiver. The precoding matrix $\mathbf{B}_{\mathrm{RF}}^{(i) \text {,DFT }}$ is built then by concatenating the beamforming vector for every user as follows:

$$
\mathbf{B}_{\mathrm{RF}}^{(i), \mathrm{DFT}}=\left[\begin{array}{llll}
\mathbf{b}_{1, i}^{\mathrm{DFT}} & \mathbf{b}_{2, i}^{\mathrm{DFT}} & \cdots & \mathbf{b}_{N_{s}, i}^{\mathrm{DFT}}
\end{array}\right]
$$

3.3. Hybrid Precoding. The best performance of hybrid precoding can be obtained by joint optimization of both $\mathbf{B}_{\mathrm{RF}}$ and $\mathbf{B}_{\mathrm{BB}}$ matrices. However, the high computational complexity of this approach for MU systems and the practical limitations imposed by the hybrid architecture motivate a separate optimization of the analog and digital stages [20, 21].

In this work, the hybrid precoding (HP) technique implemented consists of RZF precoding at baseband and a DFTbased codebook beamforming at RF. The procedure followed to compute both precoding matrices in $\mathrm{HP}$ is as follows. Once the users to be served by BS $i$ have been selected, the corresponding beamforming matrix $\mathbf{B}_{\mathrm{RF}}^{(i)}$ is computed, following the procedure described in Section 3.2. Then, the equivalent channel observed at baseband is calculated as

$$
\mathbf{H}_{\mathrm{eq}}^{(i)}=\mathbf{H}^{(i)} \mathbf{B}_{\mathrm{RF}}^{(i)} \text {. }
$$

Finally, the baseband precoding matrix $\mathbf{B}_{\mathrm{BB}}^{(i)}$ is computed by using (6), where now $\widetilde{\mathbf{B}}_{\mathrm{BB}}^{(i), \mathrm{RZF}}$ is given by

$$
\widetilde{\mathbf{B}}_{\mathrm{BB}}^{(i), \mathrm{RZF}}=\mathbf{H}_{\mathrm{eq}}^{(i)^{\dagger}}\left(\mathbf{H}_{\mathrm{eq}}^{(i)} \mathbf{H}_{\mathrm{eq}}^{(i)^{\dagger}}+\alpha \mathbf{I}\right)^{-1} .
$$

\section{Distributed Hybrid Precoding for DAS}

In this section, our proposal for distributed hybrid precoding (DHP) in DAS deployments is presented. The DHP technique is a modification of the HP scheme described in Section 3.3, which, instead of using DFT-based codebook at RF, introduces a new analog beamforming scheme called Distributed Analog Beamforming (DAB).
The reason for introducing DAB is motivated by the fact that, despite the good compromise between performance and complexity given by DFT-based beamforming in conventional systems, its application to DAS results in an inevitable performance degradation [8]. This is due to the distributed and not equally spaced antenna elements in DAS, what leads to nonorthogonal conformed beams at the RAUs and low beamforming gains. Besides, the fact that DFT beamforming vectors equally distribute the power among all the elements of the antenna array makes this beamforming technique unable to perform antenna selection schemes in DAS.

As a solution for the limitations of DFT-based beamforming in DAS, DAB proposes a simple approach to generate a codebook suitable for scenarios with distributed antennas. The main advantages of DAB are the inclusion of RAU selection, which permits each user to be served by the optimum set of RAUs, and the deletion of overlapped beams at each subarray. This approach consists of the following steps:

(i) Selecting a codebook for each RAU

(ii) Updating the selected codebook by including a null codeword that allows for the deactivation of the RAU transmission

(iii) Generating the DAB codebook by putting together all the possible combinations of codewords at the RAUs

(iv) Removing the combination of codewords that deactivate all the RAUs at the same time and normalizing the columns of the resulting codebook

These steps are explained in detail hereunder.

Let us assume that the $M$ antennas of each BS are distributed among $R$ RAUs, with $M_{R}=M / R$ being the number of equally spaced antennas per RAU. Then, in step (i), the subcodebook for every RAU is created. When DFTbased codebook is selected for each RAU $r, \mathrm{C}_{\mathrm{DFT}} \in \mathbb{C}^{M_{R} \times M_{R}}$ is created by using (8) and setting $C=M_{R}$ in order to guarantee nonoverlapping of the beams conformed at each RAU:

$$
\begin{aligned}
& \mathrm{C}_{\mathrm{DFT}}\left(M_{R} \times M_{R}\right)=\frac{1}{\sqrt{M_{R}}} \\
& \quad\left[\begin{array}{cccc}
1 & 1 & \cdots & 1 \\
1 & e^{-j 2 \pi 1\left(1 / M_{R}\right)} & \cdots & e^{-j 2 \pi 1\left(\left(M_{R}-1\right) / M_{R}\right)} \\
\vdots & \vdots & \vdots & \vdots \\
1 & e^{-j 2 \pi\left(M_{R}-1\right)\left(1 / M_{R}\right)} & \cdots & e^{-j 2 \pi\left(M_{R}-1\right)\left(\left(M_{R}-1\right) / M_{R}\right)}
\end{array}\right] .
\end{aligned}
$$

In step (ii), a null codeword is aggregated to the codebook, which allows the BS to deactivate the use of the antennas at RAU $r$ when required:

$$
\mathbf{C}_{\mathrm{DAB}}^{(r)}=\left[\begin{array}{l|l}
\mathbf{C}_{\mathrm{DFT}}\left(M_{R} \times M_{R}\right) & \vdots \\
& 0
\end{array}\right]=\left[\begin{array}{llll}
\mathbf{c}_{1} & \mathbf{c}_{2} & \cdots & \mathbf{c}_{M_{R}+1}
\end{array}\right],
$$

where $\mathbf{c}_{i} \in \mathbb{C}^{M_{R} \times 1}$ represents each column or codeword in $\mathrm{C}_{\mathrm{DAB}}^{(r)}$. 
In step (iii), a codebook containing the codewords of $M$ elements for all the antennas in the BS is generated. This step is performed by creating all the possible combinations of $R$ codewords $\mathbf{c}_{i}$ out of $\mathbf{C}_{\mathrm{DAB}}^{(r)}$. Note that there exist a total of $\left(M_{R^{+}}\right.$ $1)^{R}$ possible combinations to create the new codewords $\mathbf{m}_{i} \in$ $\mathbb{C}^{M \times 1}$.

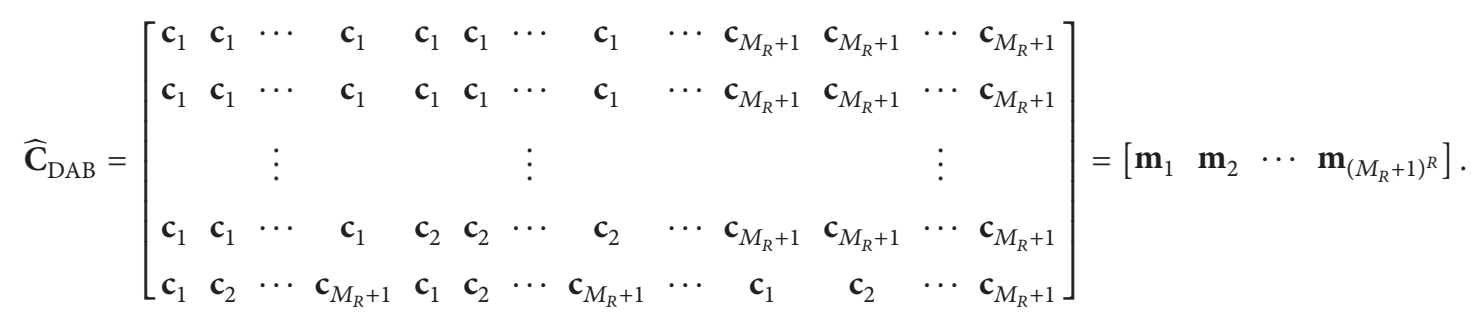

Finally, in step (iv), the last codeword $\mathbf{m}_{\left(M_{R}+1\right)^{R}}$ is removed, since it would result in the deactivation of all the RAUs at the same time. Therefore, $\mathrm{C}_{\mathrm{DAB}} \in \mathbb{C}^{M \times\left(M_{R}+1\right)^{R}-1}$ is given by

$$
\mathbf{C}_{\mathrm{DAB}}=\left[\begin{array}{llll}
\alpha_{1} \mathbf{m}_{1} & \alpha_{2} \mathbf{m}_{2} & \cdots & \alpha_{\left(M_{R}+1\right)^{R}-1} \mathbf{m}_{\left(M_{R}+1\right)^{R}-1}
\end{array}\right],
$$

where $\alpha_{i}=1 /\left|\mathbf{m}_{i}\right|, i=1, \ldots,\left(M_{R}+1\right)^{R}-1$, is applied to normalize the columns of $\widehat{\mathbf{C}}_{\mathrm{DAB}}$.

An example of DAB codebook creation is shown in Table 1 for a system with BSs equipped with 4 antennas distributed in two RAUs.

Note that, in this paper, DFT-based subcodebook has been selected for every RAU in step (i) for the sake of simplicity, although any other type of codebook could be selected instead. Indeed, DAB codebook can be built from the combination of codebooks of different types and/or sizes, what allows for its application in scenarios in which BS antennas are distributed in an irregular fashion over the cell coverage area.

Once the codebook matrix $\mathbf{C}_{\mathrm{DAB}}$ is created, the optimum beamforming vector for the $u$ th user served by BS $i$, denoted as $\mathbf{b}_{u, i}^{\mathrm{DAB}}$, is chosen in a similar way as in Section 3.2 by using now

$$
\begin{aligned}
\mathbf{b}_{u, i}^{\mathrm{DAB}} & =\alpha_{c^{*}} \mathbf{m}_{c^{*}}, \\
c^{*} & =\arg \max _{c}\left(\left|\mathbf{h}_{u, i}^{(i)^{\top}} \alpha_{c} \mathbf{m}_{c}\right|^{2}\right) .
\end{aligned}
$$

Therefore, matrix $\mathbf{B}_{\mathrm{RF}}^{(i), \mathrm{DAB}}$ is built then by concatenating the beamforming vector for every user as follows:

$$
\mathbf{B}_{\mathrm{RF}}^{(i), \mathrm{DAB}}=\left[\begin{array}{llll}
\mathbf{b}_{1, i}^{\mathrm{DAB}} & \mathbf{b}_{2, i}^{\mathrm{DAB}} & \cdots & \mathbf{b}_{N_{s}, i}^{\mathrm{DAB}}
\end{array}\right] .
$$

Finally, the procedure to compute the baseband precoding matrix $\mathbf{B}_{\mathrm{BB}}^{(i)}$ in DHP follows the same rationale as in HP, which was described in Section 3.3, although $\mathbf{B}_{\mathrm{RF}}^{(i)}$ in (12) should be replaced by (19).

\section{Simulation Setup}

This section describes the most important configuration parameters and other major considerations used for the simulation of the MIMO precoding techniques described in Sections 3 and 4. Besides, it includes a description of the indoor scenario, details about the channel model implemented, and a thorough description of the different indoor deployment strategies compared along the paper.

We consider a downlink Orthogonal Frequency Division Multiplexing (OFDM) system working in Frequency Division Duplex (FDD) mode in an indoor rectangular scenario of $120 \mathrm{~m} \times 50 \mathrm{~m}$. Total frequency bandwidth of $20 \mathrm{MHz}$ is divided in $N_{\mathrm{FB}}=100$ Frequency Blocks (FBs), each with bandwidth $\Delta f=200 \mathrm{kHz}$. The channel model used is based on the $3 \mathrm{D}$ mmWave indoor channel model proposed by Sun et al. in [22]. This model has been derived at $73 \mathrm{GHz}$, using data extracted from a propagation measurement campaign performed in a typical office scenario and filling the gaps in the measurements by means of a ray tracing tool. The model follows a 3GPP-style and characterizes the channel in azimuth, elevation, and polarization terms. Concerning large-scale fading, shadowing is added on top of the path loss term [23], with standard deviations of $1 \mathrm{~dB}$ and $9 \mathrm{~dB}$ for line-of-sight and non-line-of-sight conditions, respectively. Antenna pattern is considered to be omnidirectional.

User selection and RF precoding are done at the whole band at a time, while baseband precoding is computed per FB. The expected throughput achieved by the $u$ th user served by $\mathrm{BS} i$ is computed by using the following expression:

$$
\mathrm{Th}_{u, i}=\sum_{f=1}^{N_{\mathrm{FB}}} \Delta f \log _{2}\left(1+\operatorname{SINR}_{u, i}^{f}\right),
$$

where $\operatorname{SINR}_{u, i}^{f}$ particularizes the SINR for the FB $f$.

The performances of the different precoding schemes are compared in this indoor scenario by using three different deployment strategies [3], as depicted in Figure 2. The first strategy, labeled as CON in the figure, consists of a conventional deployment of two femtocells, each one equipped with a colocated Uniform Linear Array (ULA) of 24 elements. The second strategy is the use of regular femtocells deployments and corresponds to scenarios F4 and F8 in the figure. Since the total number of antenna elements at every scenario must be the same for the sake of a fair comparison, femtocells in F4 are equipped with 12-element ULA, while F8 femtocells have ULA of 6 elements. The last strategy is the use of DAS, 
TABLE 1: Example of DAB creation for a DAS system with BSs equipped with four antennas distributed in two RAUs.

(i) Selection of a DFT codebook suitable for each RAU.

$\mathbf{C}_{\mathrm{DFT}}^{(r)}=\left[\begin{array}{cc}1 & -1 \\ 1 & 1\end{array}\right]$

(ii) Addition of the null codeword:

$\mathbf{C}_{\mathrm{DAB}}^{(r)}=\left[\begin{array}{ccc}1 & -1 & 0 \\ 1 & 1 & 0\end{array}\right]$

(iii) Generation of codewords combination:

$\widehat{\mathbf{C}}_{\mathrm{DAB}}=\left[\begin{array}{ccccccccc}1 & 1 & 1 & -1 & -1 & -1 & 0 & 0 & 0 \\ 1 & 1 & 1 & 1 & 1 & 1 & 0 & 0 & 0 \\ 1 & -1 & 0 & 1 & -1 & 0 & 1 & -1 & 0 \\ 1 & 1 & 0 & 1 & 1 & 0 & 1 & 1 & 0\end{array}\right]$

(iv) Deletion of null codeword and normalization of every codeword:

$\mathrm{C}_{\mathrm{DAB}}=\left[\begin{array}{cccccccc}\frac{1}{2} & \frac{1}{2} & \frac{1}{\sqrt{2}} & -\frac{1}{2} & -\frac{1}{2} & -\frac{1}{\sqrt{2}} & 0 & 0 \\ \frac{1}{2} & \frac{1}{2} & \frac{1}{\sqrt{2}} & \frac{1}{2} & \frac{1}{2} & \frac{1}{\sqrt{2}} & 0 & 0 \\ \frac{1}{2} & -\frac{1}{2} & 0 & \frac{1}{2} & -\frac{1}{2} & 0 & \frac{1}{\sqrt{2}} & -\frac{1}{\sqrt{2}} \\ \frac{1}{2} & \frac{1}{2} & 0 & \frac{1}{2} & \frac{1}{2} & 0 & \frac{1}{\sqrt{2}} & \frac{1}{\sqrt{2}}\end{array}\right]$

considering a deployment with two cells, each one equipped with 24 antenna elements. However, in this case, antennas are not colocated but are distributed among several RAUs, as depicted in scenarios DAS4 and DAS8. In DAS4, each cell has 2 RAUs equipped with a ULA of 12 elements, while in DAS8 each cell has 4 RAUs equipped with a ULA of 6 elements. The summary of the configuration details for each scenario is given in Table 2 .

For the sake of a fair comparison among the defined scenarios, a site is supposed to be composed in every deployment by the set of cells located in a half of the scenario, considering a vertical dividing line located at the center of it. Therefore, for CON, DAS4, and DAS8 scenarios, a site corresponds to a cell, while for F4 and F8 deployments a site is composed by two and four femtocells, respectively. Note that, using this definition, the power and number of antennas per site are exactly the same for all the deployments.

Although no coordination among BSs is considered, joint transmission from all the RAUs belonging to a BS is assumed in DAS deployments. Moreover, the wires used to connect the distributed antennas with the BSs are assumed to be ideal in all the simulations. Other important parameters used for the simulations are collected in Table 3.

\section{Results and Discussion}

In this section, the scenarios defined in Section 5 are evaluated and compared under different assumptions. Firstly, the three deployment strategies are analyzed by means of the defined scenarios when using fully digital and hybrid precoding and assuming ideal conditions. Afterwards, the same scenarios are analyzed including the effect of nonideal assumptions such as outdated CSI or losses introduced by nonideal RF combiners.

6.1. Performance Comparison of Ideal Indoor Deployment Strategies. In this subsection, a performance comparison of the different indoor deployment strategies is conducted for both fully digital and hybrid precoding under ideal assumptions. Although fully digital precoding schemes are not suitable for mmWave systems with large antenna arrays, their simulation can provide us with an upper bound of performance for the evaluation of the hybrid implemented schemes. For that reason, simulation results with RZF precoding are presented in the first place, and the performance of HP and DHP in the different deployments is analyzed afterwards. Finally, a brief comparison between RZF and $\mathrm{DHP}$ is performed focusing on one specific scenario.

6.1.1. Results Using Fully Digital Precoding. For the simulation of RZF precoding in the different deployments, each BS is considered to be equipped with as many RF chains as antennas, what results in a total of $48 \mathrm{RF}$ chains in the system.

The average number of multiplexed users per site is collected in Table 4 for all the simulated scenarios. RZF makes the most of the available RF chains and is able to multiplex all possible users in the site. Only a small loss in the number of multiplexed users is seen for F8, given by a small imbalance among the amount of users assigned to each femtocell, which will disappear with the average of more simulation drops.

Figure 3 shows the Cumulative Distribution Function (CDF) of the user spectral efficiency when RZF precoding is applied. As expected, user spectral efficiency values are mostly smaller for those deployments with lower degree of coordination, such as F4 and F8. Note that in F8 only up to 6 users can be multiplexed simultaneously avoiding interuser interference, while in CON or DAS deployments, up to 24 users are multiplexed with no intracell interference. On the other hand, the difference between CON and DAS is related to the antenna elements location. While in DAS deployments the antennas are distributed over the space, bringing a larger benefit to those users located on the cell edges, in CON deployment all the antenna elements are colocated at the cell center, providing higher beamforming gains that benefit those users located near the BS. Indeed, increasing the number of coordinated antennas enhances the user spectral efficiency, while distributing the antennas over the space improves the user fairness. These results point to a very interesting conclusion in good consonance with the results discussed in [24], indicating that DAS deployments bring about a significant gain in terms of average user rate and cell-edge user rate at the cost of reducing the system peak rate.

The CDF of the system throughput is depicted in Figure 4. This figure highlights that the cell throughput distributions for different options of DAS and CON are much more similar in comparison with the user distributions. This behavior is basically caused by the above-mentioned peak effect. Indeed, the more the antenna elements are distributed, provided that interferences are coordinated, the better it is for the fairness 
TABLE 2: Configuration details of the simulated scenarios, where $M$ is the number of antennas per BS, $R$ is the number of RAUs per BS, and $M_{R}$ is the number of antennas per RAU.

\begin{tabular}{lcccccc}
\hline & Number of BSs & $R$ & $P$ & $P_{\text {RAU }}$ & $M$ & $M_{R}$ \\
\hline CON & 2 & - & $30 \mathrm{dBm}$ & - & 24 & - \\
F4 & 4 & - & $27 \mathrm{dBm}$ & - & 12 & - \\
F8 & 8 & - & $24 \mathrm{dBm}$ & - & 6 & - \\
DAS4 & 2 & 2 & $30 \mathrm{dBm}$ & $27 \mathrm{dBm}$ & 24 & 12 \\
DAS8 & 2 & 4 & $30 \mathrm{dBm}$ & $24 \mathrm{dBm}$ & 24 & 6 \\
\hline
\end{tabular}
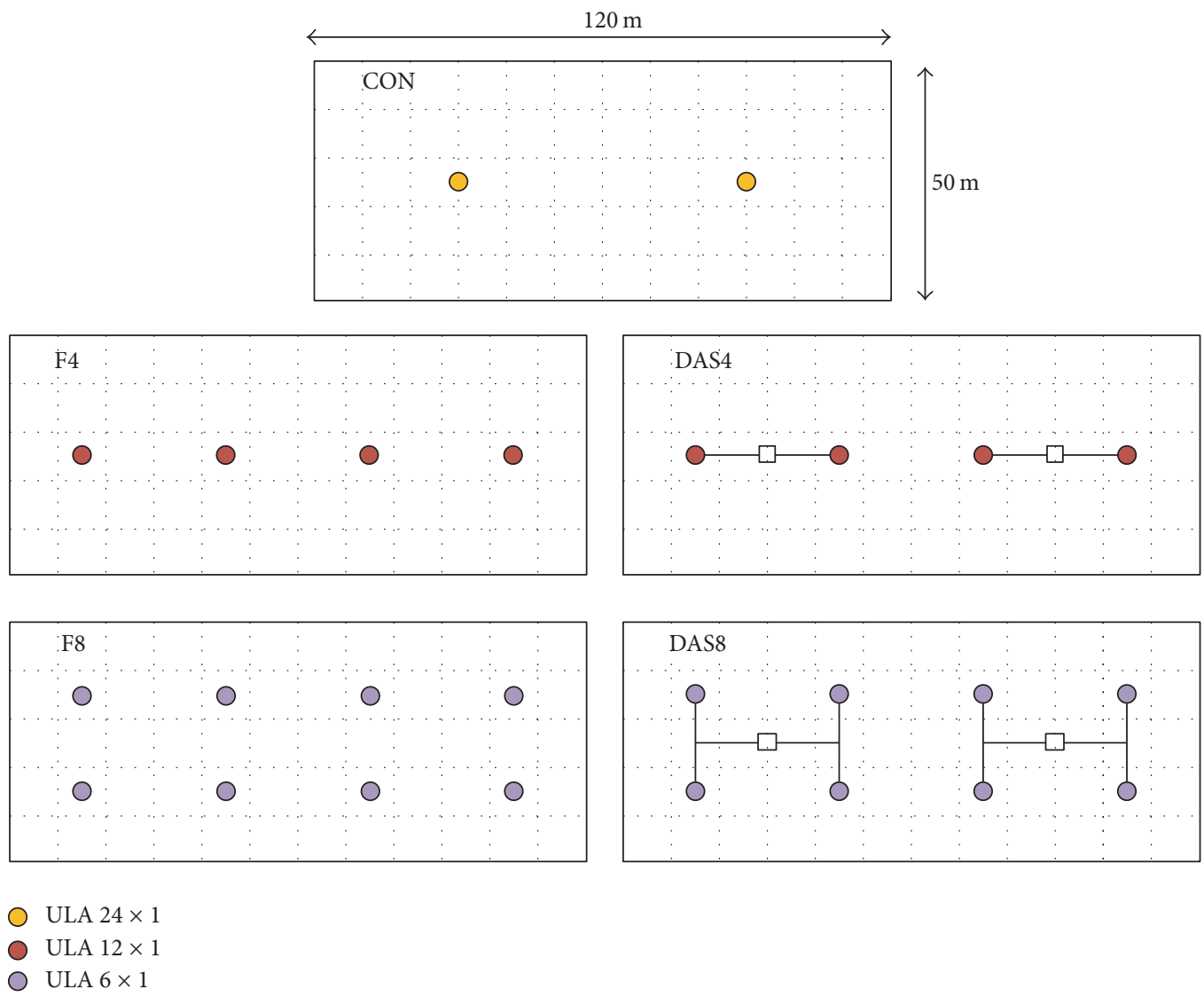

FIGURE 2: Scenarios defined for the comparison of different indoor deployment strategies, including a conventional deployment with two cells (CON), two femtocell deployments with 4 and 8 cells (F4 and F8), and DAS with 4 and 8 RAUs (DAS4 and DAS8).

in the system, but this comes at the expense of reducing the data rate of users in best radio conditions, and the average effect in the system is not that huge. Still, there is a big gap in performance between the femtocell deployments and the other options with coordinated interferences, such as CON or DAS.

Finally, Table 5 collects the comparison of some network performance indicators, including also the gains achieved by the different deployments with respect to the conventional deployment. Approximately same average values of user rate and site rate are achieved by CON and DAS, while the best 5th percentile of user rate is reached by DAS8. The huge improvement in terms of cell-edge user rate achieved by DAS deployments is worth noting, which can outperform the conventional deployment by a factor of 6.
6.1.2. Results Using Hybrid Precoding. Simulation results considering hybrid precoding are hereafter presented. For all the scenarios, HP is applied by performing RZF at baseband and DFT-based precoding at RF. Besides, for DAS deployments, also DHP described in Section 4 is used to better exploit the distributed nature of the system, which consists in the application of RZF and DAB at baseband and RF stages, respectively. In all the scenarios, the number of RF chains is limited to 8 per site. The choice of this number of RF chains is motivated by current limitations of BSs.

Table 6 shows the average number of simultaneous multiplexed users per site for all the considered scenarios when using hybrid precoding. Taking into account the fact that the maximum number of multiplexed users is limited by the available RF chains, it can be observed that almost all the algorithms harness the multiplexing gain. Only DAS8 
TABLE 3: Simulation parameters.

\begin{tabular}{lc}
\hline Simulation time per drop & $1 \mathrm{~s}$ \\
Number of drops & 10 \\
Subframe duration, $T_{s}$ & $1 \mathrm{~ms}$ \\
Carrier frequency & $73 \mathrm{GHz}$ \\
Number of FBs, $N_{\mathrm{FB}}$ & 100 \\
FB bandwidth, $\Delta f$ & $200 \mathrm{kHz}$ \\
Scheduling policy & Round-robin \\
Thermal noise PSD & $-174 \mathrm{dBm} / \mathrm{Hz}$ \\
BS height & $6 \mathrm{~m}$ \\
BS antenna element pattern & Omnidirectional \\
Number of deployed UEs & 100 \\
UE antenna element pattern & Omnidirectional \\
UE noise figure & $7 \mathrm{~dB}$ \\
UE speed & $3 \mathrm{~km} / \mathrm{h}$ \\
UE height & $1.5 \mathrm{~m}$ \\
\hline
\end{tabular}

TABLE 4: Average number of multiplexed users per site with RZF for every simulated scenario.

$$
\text { CON RZF DAS4 RZF F4 RZF DAS8 RZF F8 RZF }
$$

Avg.

multiplexed $24.00 \quad 24.00 \quad 24.00^{*} \quad 24.00 \quad 23.95^{*}$

users/site

${ }^{*}$ In this case, a site is assumed to be the group of cells located in half the scenario.

with HP shows a lower number of multiplexed users in comparison to the others.

User spectral efficiency is depicted in Figure 5, where two different areas can be distinguished. On one hand, at the lower part of the CDF, femtocell deployments show the best performance, followed by DAS systems using DHP. This is explained by the antennas distribution, which improves the rates delivered to cell-edge users. Note, however, that DAS deployments using HP do not provide this rate gain to celledge users, due to the intracell interference created by the overlap of the beams conformed by HP and the lack of RAU selection of the algorithm. On the other hand, at the upper part of the CDF, femtocell deployments provide the poorest performance among all the algorithms. Indeed, limiting the number of RF chains greatly affects conventional systems and, therefore, there is a need to distribute the transmitters in some way, increasing the deployment density. Compared to a denser femtocell solution, DAS deployments offer practically the same performance as dense femtocells for users in worse radio conditions, while ostensibly improving the quality of the users in good radio conditions, which has a much greater impact on cell rate.

In terms of system performance, curves in Figure 6 show that the application of DHP in DAS brings about a large increase of the system throughput with respect to the use of HP. Also, the use of DAS is shown to be by far the best indoor deployment and, in general, the distribution of the antennas over the scenario is beneficial. Having a look at the network performance indicators collected in Table 7, enormous gains

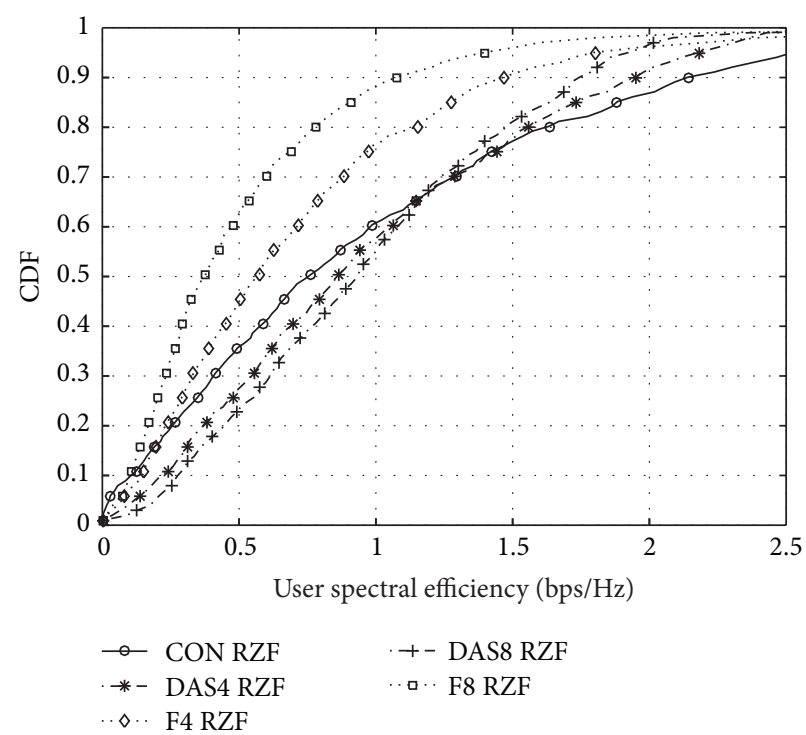

FIGURE 3: User spectral efficiency's CDF comparison using RZF precoding for all the simulated scenarios.

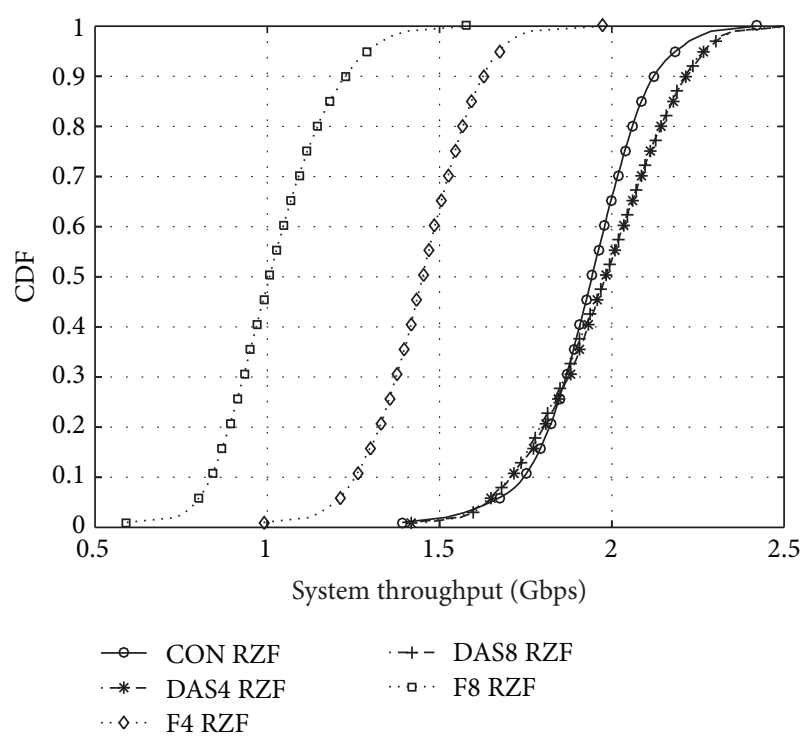

FIGURE 4: System throughput's CDF comparison using RZF precoding for all the simulated scenarios.

in 5th percentile user rate for DAS and femtocell deployments can be observed with respect to the conventional scenario.

Finally, a brief comparison between the performances of DAS8 scenario using RZF and DHP is presented in Table 8. The use of hybrid architectures implies a loss in average user rate and site rate around the $12 \%$ with respect to the digital precoding, mostly brought about by the reduction in the RF chains which, especially for this simulation study, has been reduced from a total of 48 to 16 .

6.2. Performance Comparison of Nonideal Indoor Deployment Strategies. The results presented in the previous section assumed ideal conditions for all the components in the 
TABLE 5: Comparison of average performance indicators with RZF.

\begin{tabular}{|c|c|c|c|c|c|}
\hline & CON RZF & DAS4 RZF & F4 RZF & DAS8 RZF & F8 RZF \\
\hline Avg. user rate [Mbps] & 19.39 & 19.75 & 14.49 & 19.74 & 10.22 \\
\hline Gain [\%] & - & 1.86 & -25.29 & 1.78 & -47.28 \\
\hline 5th percentile user rate [Mbps] & 0.58 & 2.70 & 1.66 & 4.10 & 1.39 \\
\hline Gain [\%] & - & 366.76 & 186.56 & 609.09 & 140.89 \\
\hline Avg. site rate [Gbps] & 0.97 & 0.99 & $0.72^{*}$ & 0.99 & $0.51^{*}$ \\
\hline Gain [\%] & - & 1.86 & -25.29 & 1.78 & -47.28 \\
\hline
\end{tabular}

${ }^{*}$ In this case, a site is assumed to be the group of cells located in half the scenario.

TABLE 6: Average number of multiplexed users per site with HP and DHP for every simulated scenario.

\begin{tabular}{lccccccc}
\hline & CON & DAS4 & DAS4 & F4 & DAS8 & DAS8 & F8 \\
& HP & HP & DHP & HP & HP & DHP & HP \\
\hline Avg. multiplexed users/site & 7.69 & 7.86 & 7.93 & $7.85^{*}$ & 6.72 & 7.72 & $7.39^{*}$ \\
\hline
\end{tabular}

${ }^{*}$ In this case, a site is assumed to be the group of cells located in half the scenario.

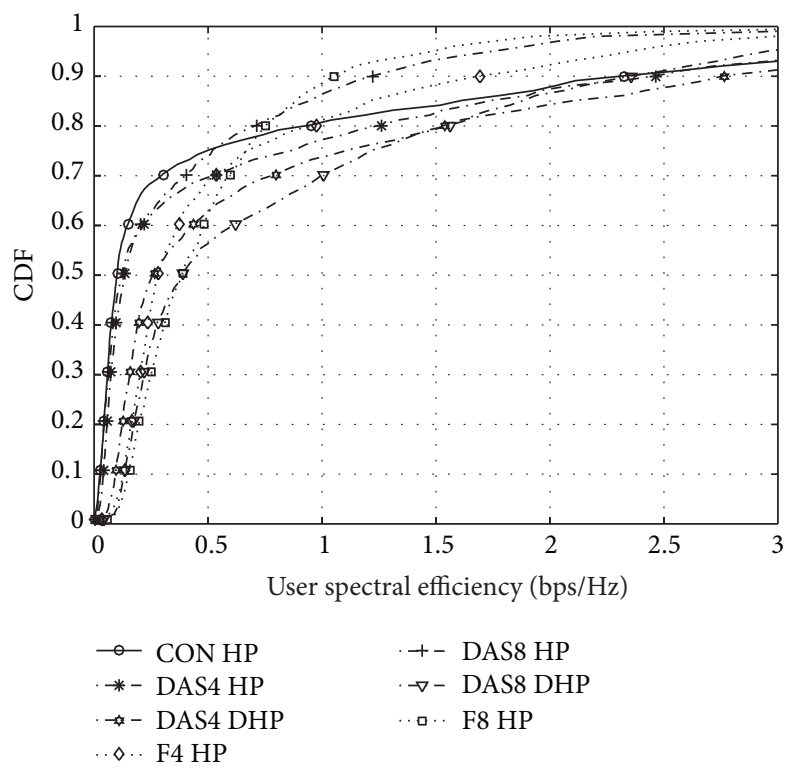

FIGURE 5: User spectral efficiency's CDF comparison using hybrid precoding for all the considered deployments.

system. However, in real systems, the presence of practical limitations or hardware impairments may significantly deteriorate the system's performance. For that reason, a performance evaluation is presented in this section including two nonideal phenomena with high impact in hybrid architectures: the use of outdated CSI at the transmitter and the losses introduced by real combiners.

6.2.1. Outdated CSI. The beamforming and multiplexing gains obtained by using multiuser transmission have been shown to be very sensitive to inaccuracies of the CSI at the transmitter. Indeed, in FDD systems, where channel state is estimated at the receiver and fed back to the transmitter afterwards, the delay between the time of measurement and use can make the CSI used at the transmitter be already outdated. Taking into account this practical issue, the impact

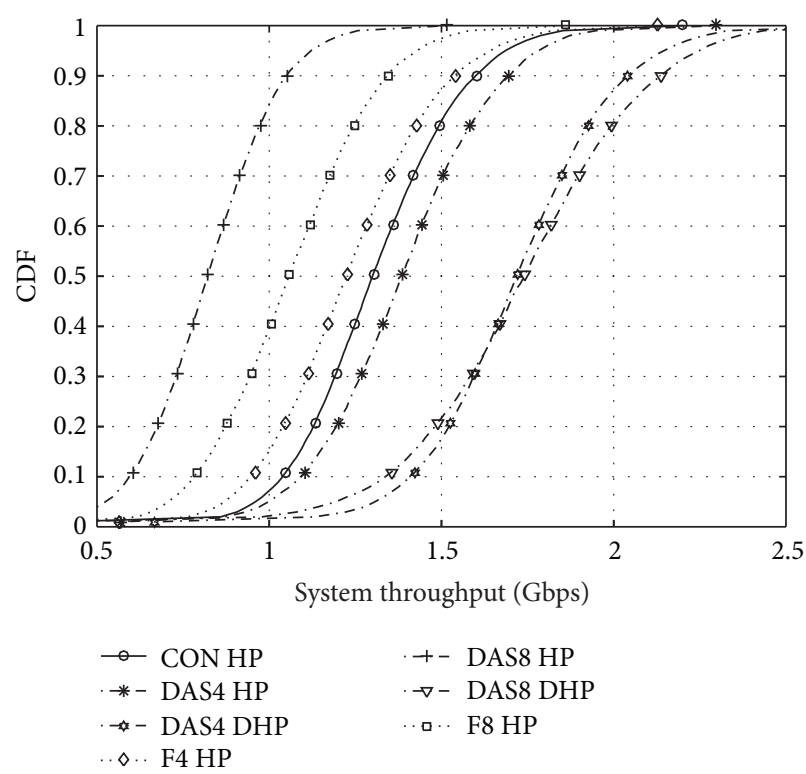

FIGURE 6: System throughput's CDF comparison using hybrid precoding for all the considered deployments.

of outdated CSI in the hybrid precoding schemes is analyzed in this section for all the deployments described in Section 5. To this aim, hybrid schemes have been simulated considering that each BS receives an update of the channel coefficients only every $T \mathrm{~ms}$, with $T$ being greater than the subframe duration $T_{s}$.

In particular, Figure 7 represents the CDF of the user spectral efficiency when $T=10 \mathrm{~ms}$ in comparison with the ideal case of timely CSI $\left(T=T_{s}=1\right)$. It is easily observed that the outdated CSI reduces the user spectral efficiency in all cases, but the degradation is particularly significant for those users with better channel conditions, that is, the users with higher spectral efficiencies.

Average user rate values are depicted in Figure 8 for different values of $T$. Note that, despite the fact that the DAS deployments show a better performance when perfect 
TABLE 7: Comparison of average performance indicator values for indoor deployments using hybrid precoding.

\begin{tabular}{|c|c|c|c|c|c|c|c|}
\hline & $\mathrm{CON}$ & DAS4 & DAS4 & $\mathrm{F} 4$ & DAS8 & DAS8 & F8 \\
\hline & HP & HP & DHP & HP & HP & DHP & HP \\
\hline Avg. user rate [Mbps] & 13.15 & 13.93 & 17.27 & 12.40 & 8.27 & 17.42 & 10.66 \\
\hline Gain $[\%]$ & - & +5.91 & +31.38 & -5.68 & -37.14 & +32.45 & -18.90 \\
\hline 5th percentile user rate [Mbps] & 0.35 & 0.64 & 1.53 & 2.14 & 0.40 & 2.29 & 2.59 \\
\hline Gain [\%] & - & +80.63 & +332.82 & +504.75 & +13.27 & +547.44 & +629.30 \\
\hline Avg. site rate [Gbps] & 0.66 & 0.70 & 0.86 & $0.62^{*}$ & 0.41 & 0.87 & $0.53^{*}$ \\
\hline Gain [\%] & - & +5.91 & +31.38 & -5.68 & -37.14 & +32.45 & -18.90 \\
\hline
\end{tabular}

${ }^{*}$ In this case, a site is assumed to be the group of cells located in half the scenario.

TABLE 8: Comparison of average values.

\begin{tabular}{lcc}
\hline & DAS8 & DAS8 \\
& RZF & DHP \\
\hline Avg. user rate [Mbps] & 19.74 & 17.42 \\
Gain [\%] & 0.00 & -11.77 \\
5th percentile user rate [Mbps] & 4.10 & 2.29 \\
Gain [\%] & 0.00 & -44.01 \\
Avg. site rate [Gbps] & 0.99 & 0.87 \\
Gain [\%] & 0.00 & -11.77 \\
Avg. scheduled users/site & 24.00 & 7.72 \\
\hline
\end{tabular}

CSI is available at the transmitter, they are more sensitive to outdated CSI than femtocell deployments. This can be explained by the size of the channel vector used in the scheduling decisions, which is of 24 elements in DAS4 and DAS8 deployments (the number of all the antennas belonging to the same cell) and only of 12 and 6 elements in F4 and F8, respectively. Indeed, the most robust deployment to outdated CSI is shown to be F8, which overcomes the performance of the other deployments for $T \geq 5 \mathrm{~ms}$.

6.2.2. Combiner Losses. In the hybrid architecture shown in Figure 1, the signals of the different RF chains are mixed together by means of a combiner to feed the antenna array. Real combiners introduce a power loss in their outputs [25], which is shown to be of great importance in the system design, since losses escalate with the number of branches to be combined [14].

Let us denote by $L$ the power loss of a basic combiner with two input branches and assume that a combiner for a generic number $P$ of RF chains is implemented by means of a cascade of $\log _{2} P$ two-branch combiners, leading to a total loss, expressed in logarithmic units of

$$
L^{\text {(tot) }}[\mathrm{dB}]=\log _{2} P \cdot L[\mathrm{~dB}] .
$$

The impact of the combiner losses on the performance of the hybrid schemes is analyzed for the three different deployment strategies by setting $L=1,2$, and $3 \mathrm{~dB}$ and comparison with the case of ideal combiners $(L=0 \mathrm{~dB})$. Note that the number of required combiners differs from one scenario to the other. For instance, in DAS4 scenario, each BS has 8 RF chains; hence signals pass through 3 combiners

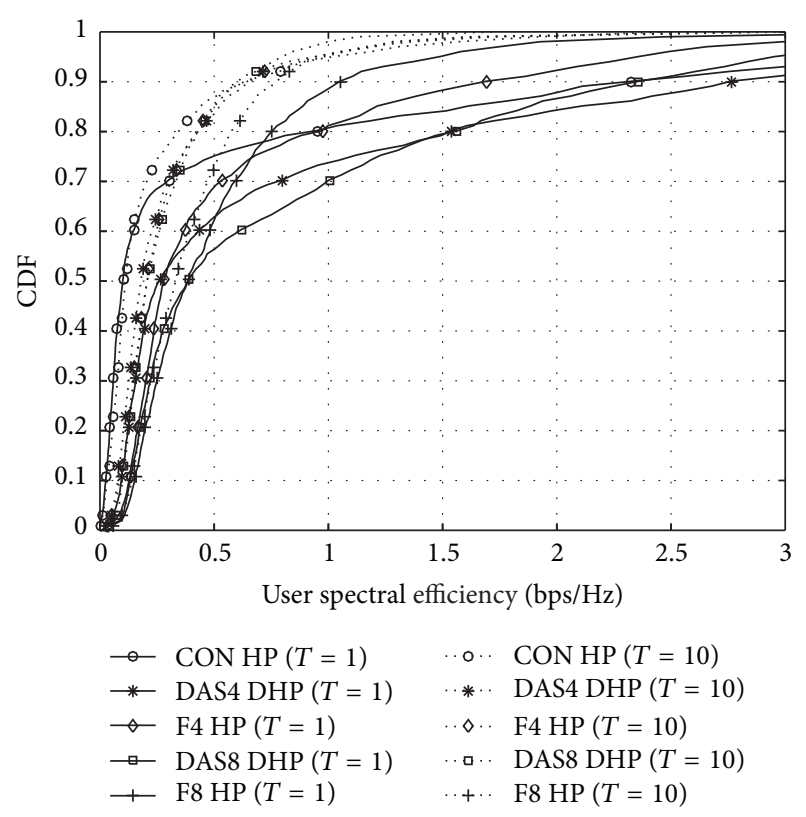

FIGURE 7: CDFs of the user spectral efficiency with outdated CSI.

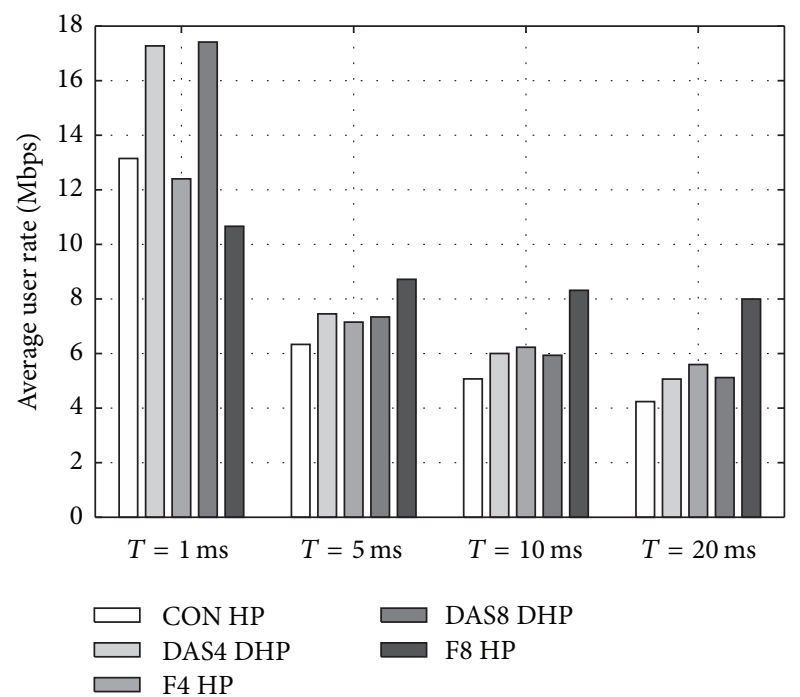

FIGURE 8: Average user rate with outdated CSI. 


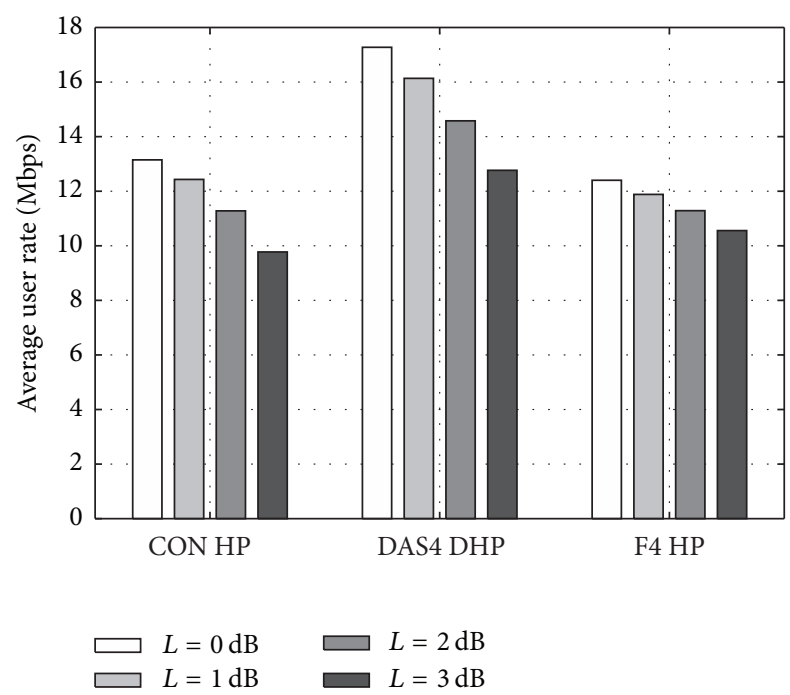

FIGURE 9: Average user data rate achieved by hybrid schemes when considering combiner losses.

before reaching the antenna array, while in F4 scenario there are $4 \mathrm{RF}$ chains per femtocell and only 2 combiners are required to distribute signals.

Figure 9 shows the average user rate achieved by CON, DAS4, and F4 as a function of the parameter L. Although DAS is the deployment option most affected by combiner losses, it remains the solution providing the highest average user rates even for the largest value of $L$.

The CDF of the system throughput is represented in Figure 10 for $L=0$ and $3 \mathrm{~dB}$. Here, the effectiveness of DAS deployments using DHP is demonstrated and it results in the fact that the DAS4 DHP option with large combiner losses is still comparable to the other two deployment strategies under ideal conditions.

\section{Conclusion}

This paper presents a performance analysis of a distributed hybrid precoding algorithm with RAU selection capabilities for indoor DAS working in the mmWave frequency band. It further provides its comparison with two other indoor deployment strategies: a conventional deployment with two BSs and large colocated antenna arrays and a regular femtocell deployment.

System level simulations were firstly conducted considering MU MIMO digital and hybrid precoding schemes under ideal conditions for the three deployment strategies. Simulation results showed that DAS presents the best overall performance when using the proposed DHP technique, while femtocell deployments provide slightly higher data rates for cell-edge users. Regarding the comparison between hybrid and fully digital schemes, it is shown that, by reducing the number of RF chains to one-third, the system throughput only decreases about $12 \%$ and this affects mostly the users in the cell edge.

Furthermore, two important practical limitations identified in hybrid architectures were analyzed for the aforementioned scenarios. Specifically, hybrid schemes were simulated

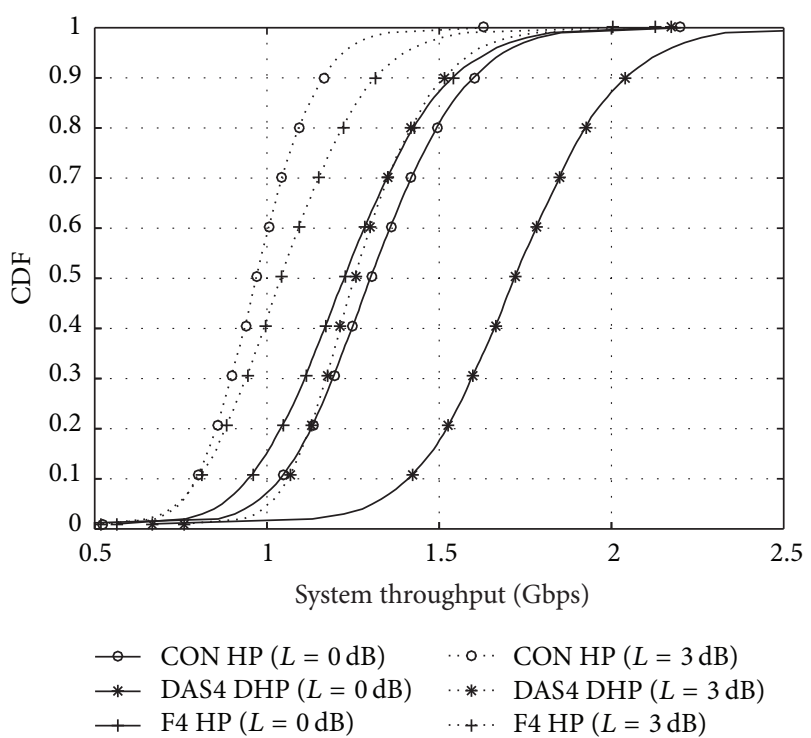

FIGURE 10: System throughput's CDF of the hybrid schemes when considering combiner losses.

considering outdated CSI at the transmitter and losses introduced by real combiners. After this analysis, DAS are shown to be very sensitive to inaccuracies in the CSI; thus, femtocell deployments are a more suitable option when the CSI update period is greater than $5 \mathrm{~ms}$. On the other hand, results demonstrate that DAS deployments are less affected by the losses introduced by the combiners and keep the best performance in comparison to the rest of strategies even for large combiner losses values.

\section{Conflicts of Interest}

The authors declare that they have no conflicts of interest.

\section{Acknowledgments}

This work has been supported by Ministerio de Economia y Competitividad, Spain (BES-2012-055975 and TEC201460258-C2-1-R), and by the European FEDER funds.

\section{References}

[1] C.-X. Wang, F. Haider, X. Gao et al., "Cellular architecture and key technologies for $5 \mathrm{G}$ wireless communication networks," IEEE Communications Magazine, vol. 52, no. 2, pp. 122-130, 2014.

[2] J. G. Andrews, S. Buzzi, and W. Choi, "What will 5G be?" IEEE Journal on Selected Areas in Communications, vol. 32, no. 6, pp. 1065-1082, 2014.

[3] T. V. Krishnamurthy and R. Shetty, 4G: Deployment Strategies and Operational Implications, Apress, Berkeley, CA, 2014.

[4] R. Heath, S. Peters, Y. Wang, and J. Zhang, "A current perspective on distributed antenna systems for the downlink of cellular systems," IEEE Communications Magazine, vol. 51, no. 4, pp. 161167, 2013. 
[5] W. Choi and J. G. Andrews, "Downlink performance and capacity of distributed antenna systems in a multicell environment," IEEE Transactions on Wireless Communications, vol. 6, no. 1, pp. 69-73, 2007.

[6] M. Tolstrup, "Indoor Radio Planning: A Practical Guide for GSM, DCS, UMTS, HSPA and LTE,' Indoor Radio Planning: A Practical Guide for GSM, DCS, UMTS, HSPA and LTE, 2011.

[7] N. Petrovic and D. Savkovic, "LTE performance in a hybrid indoor das (Active vs. Passive)," in Proceedings of the 23rd Telecommunications Forum, TELFOR 2015, pp. 141-144, srb, November 2015.

[8] X. Chen, Z. Zhang, and H.-H. Chen, “On distributed antenna systems with limited feedback precoding: opportunities and challenges," IEEE Wireless Communications, vol. 17, no. 2, pp. 80-88, 2010.

[9] S. Schwarz, R. W. Heath Jr., and M. Rupp, "Single-user MIMO versus multi-user MIMO in distributed antenna systems with limited feedback," EURASIP Journal on Advances in Signal Processing, vol. 2013, article 54, 2013.

[10] T. Rappaport, S. Sun, R. Mayzus et al., "Millimeter wave mobile communications for 5G cellular: it will work!," IEEE Access, vol. 1, pp. 335-349, 2013.

[11] R. W. Heath, N. Gonzalez-Prelcic, S. Rangan, W. Roh, and A. M. Sayeed, "An overview of signal processing techniques for millimeter wave MIMO systems," IEEE Journal of Selected Topics in Signal Processing, vol. 10, no. 3, pp. 436-453, 2016.

[12] R. Mendez-Rial, C. Rusu, N. Gonzalez-Prelcic, A. Alkhateeb, and R. W. Heath, "Hybrid MIMO Architectures for Millimeter Wave Communications: Phase Shifters or Switches?" IEEE Access, vol. 4, pp. 247-267, 2016.

[13] N. Chen, S. Sun, M. Kadoch, and B. Rong, "SDN controlled mmwave massive MIMO hybrid precoding for $5 \mathrm{G}$ heterogeneous mobile systems," Mobile Information Systems, vol. 2016, Article ID 9767065, 10 pages, 2016.

[14] S. Gimenez, S. Roger, P. Baracca et al., "Performance evaluation of analog beamforming with hardware impairments for $\mathrm{mmW}$ massive MIMO communication in an urban scenario," Sensors (Switzerland), vol. 16, no. 10, article no. 1555, 2016.

[15] J. Zhou and J. Thompson, "Linear precoding for the downlink of multiple input single output coexisting wireless systems," IET Communications, vol. 2, no. 6, pp. 742-752, 2008.

[16] A. Wiesel, Y. C. Eldar, and S. Shamai, "Zero-forcing precoding and generalized inverses," IEEE Transactions on Signal Processing, vol. 56, no. 9, pp. 4409-4418, 2008.

[17] C. B. Peel, B. M. Hochwald, and A. L. Swindlehurst, "A vector-perturbation technique for near-capacity multiantenna multiuser communication-part I: channel inversion and regularization," IEEE Transactions on Communications, vol. 53, no. 1, pp. 195-202, 2005.

[18] Y. T. Wu, Y. Y. Zhao, and F. Yu, "Comparison of codebooks for beamforming in limited feedback MIMO systems," in Proceedings of the 2012 IEEE International Conference on Computer Science and Automation Engineering, CSAE 2012, pp. 32-36, chn, May 2012.

[19] D. Yang, L.-L. Yang, and L. Hanzo, "DFT-based beamforming weight-vector codebook design for spatially correlated channels in the unitary precoding aided multiuser downlink," in Proceedings of the IEEE International Conference on Communications (ICC '10), pp. 1-5, May 2010.

[20] C. Rusu, R. Mendez-Rial, N. Gonzalez-Prelcic, and R. W. Heath, "Low Complexity Hybrid Precoding Strategies for Millimeter
Wave Communication Systems," IEEE Transactions on Wireless Communications, vol. 15, no. 12, pp. 8380-8393, 2016.

[21] F. Sohrabi and W. Yu, "Hybrid digital and analog beamforming design for large-scale antenna arrays," IEEE Journal on Selected Topics in Signal Processing, vol. 10, no. 3, pp. 501-513, 2016.

[22] S. Sun, T. S. Rappaport, T. A. Thomas, and A. Ghosh, "A preliminary 3D mm wave indoor office channel model," in Proceedings of the 2015 International Conference on Computing, Networking and Communications, ICNC 2015, pp. 26-31, usa, February 2015.

[23] J. F. Monserrat, R. Fraile, and L. Rubio, "Application of alternating projection method to ensure feasibility of shadowing crosscorrelation models," Electronics Letters, vol. 43, no. 13, pp. 724725, 2007.

[24] X.-H. You, D.-M. Wang, B. Sheng, X.-Q. Gao, X.-S. Zhao, and M. Chen, "Cooperative distributed antenna systems for mobile communications," IEEE Wireless Communications, vol. 17, no. 3, pp. 35-43, 2010.

[25] D. M. Pozar, Microwave Engineering, vol. 7, John Wiley and Sons, Inc., 2012. 

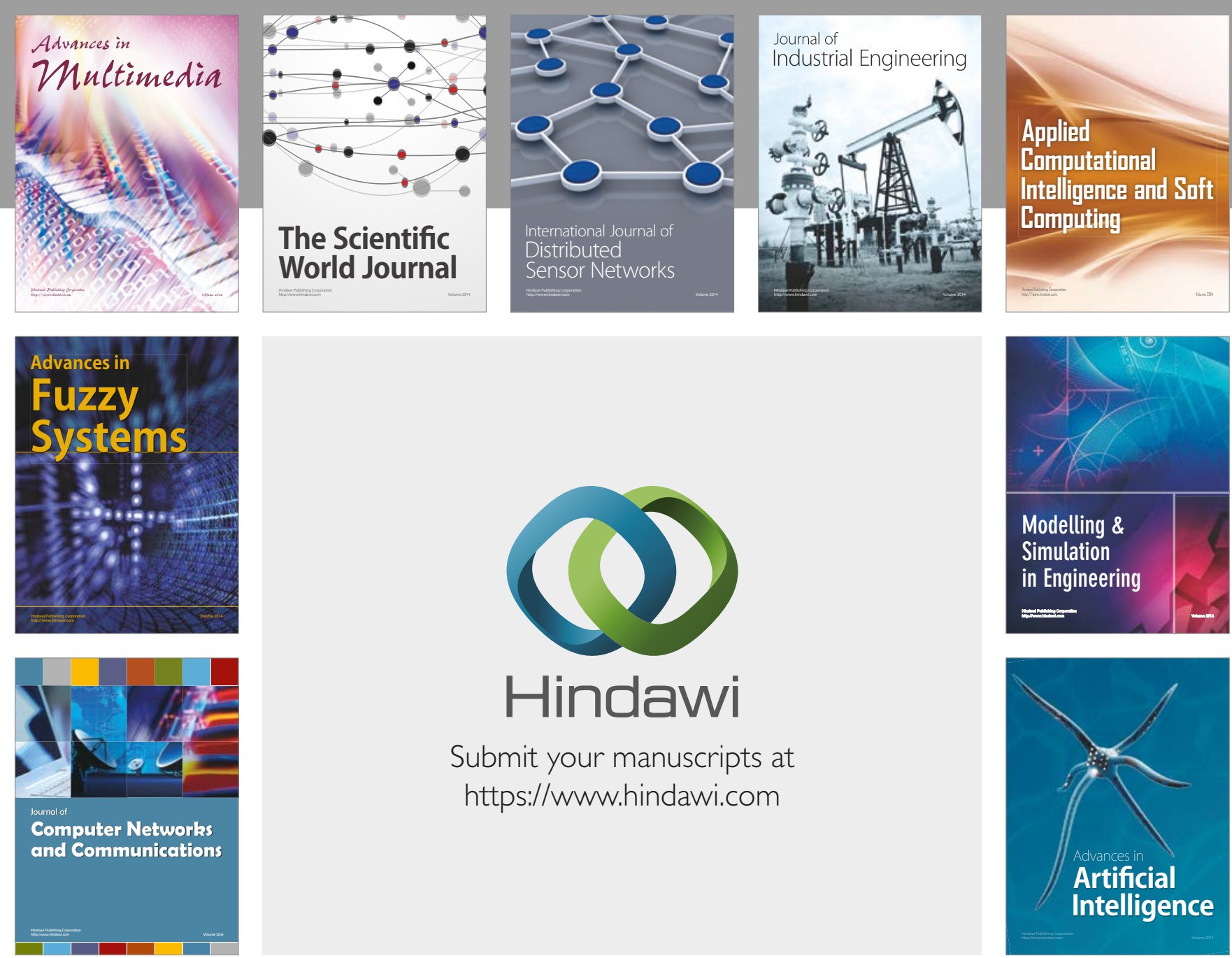

\section{Hindawi}

Submit your manuscripts at

https://www.hindawi.com
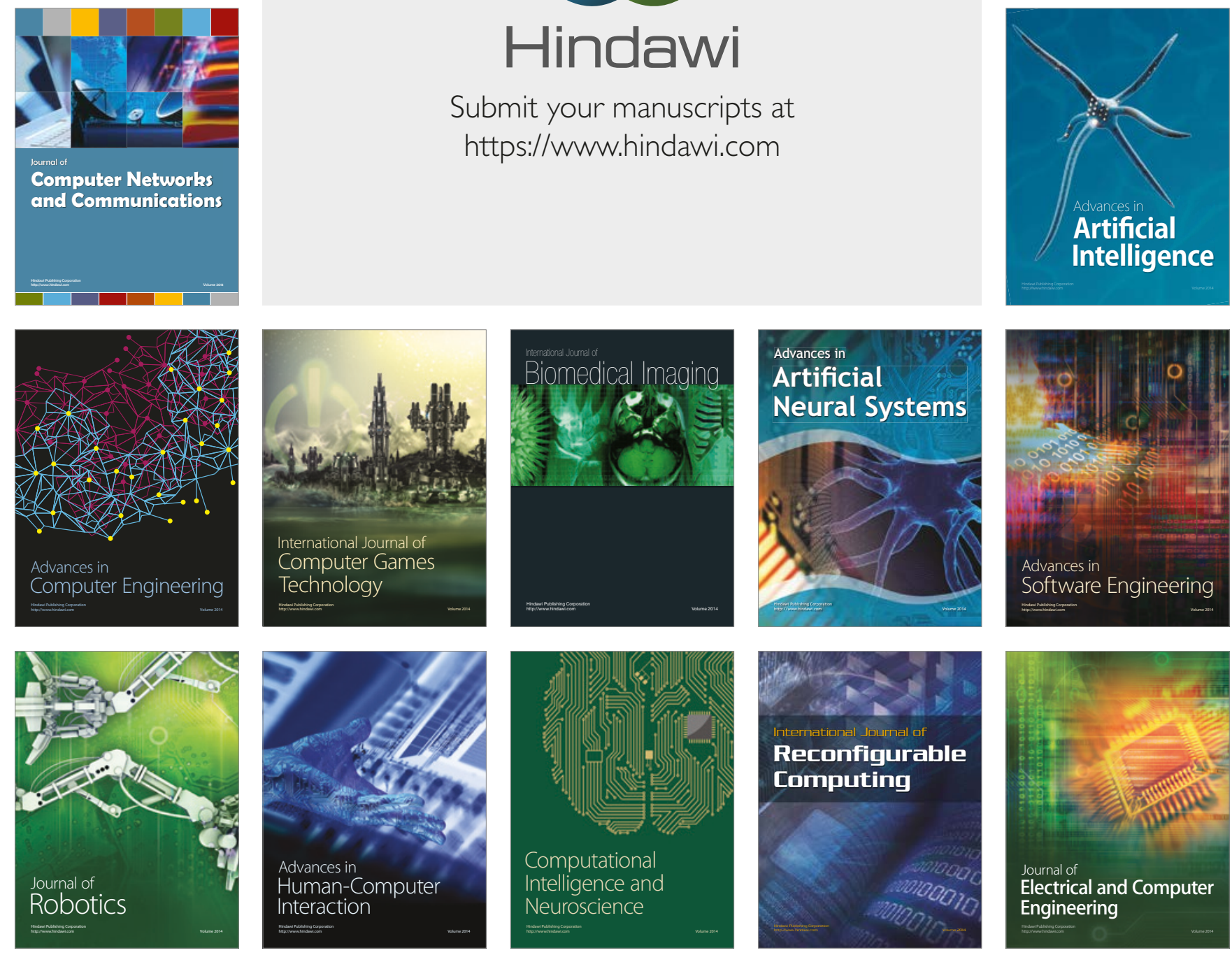